\title{
Invasive management of deep vein thrombosis
}

\author{
Nigel I Jowett, Charles C F Robinson, William M Clow
}

A 35-year-old woman presented with a two-week history of pain in the left hip. A non-smoker, she had been taking low-dose oral contraception (Logynon, Schering) for two years. There was no gynaecological symptomatology, but she had gained $5 \mathrm{~kg}$ bodyweight over 12-18 months, despite dieting. The appearance of the left leg suggested deep vein thrombosis, and venography demonstrated extensive thrombosis extending from the left femoral vein to the common iliac vein. The right iliac system was clear. Further imaging was performed (figures 1 and 2).
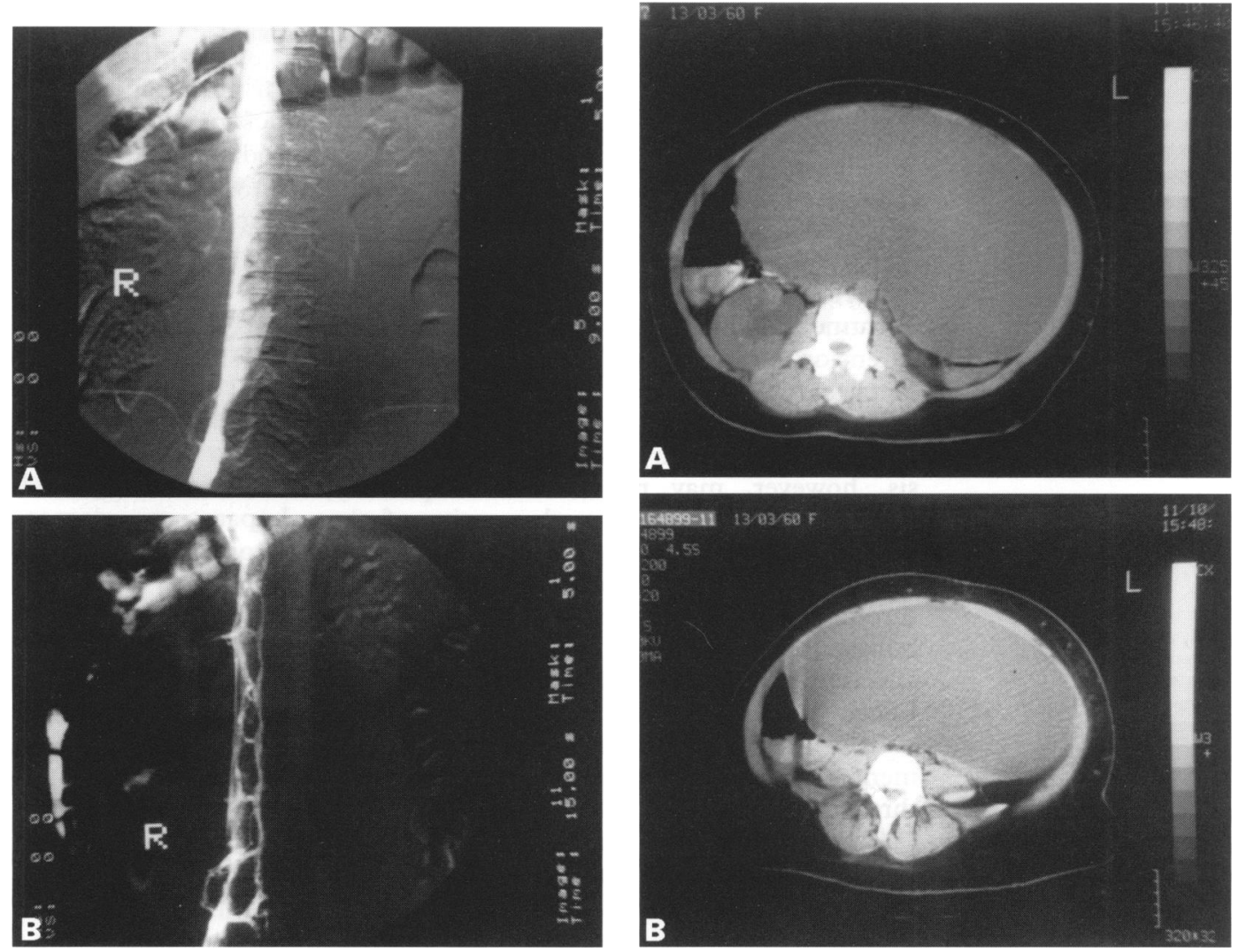

Figure 1 Digital subtraction images of an inferior

Withybush General cavagram

Figure 2 Two cuts of an abdominal CT scan

\section{Hospital,}

Haverfordwest,

Pembrokeshire, Wales

SA61 2PZ, UK

N I Jowett

C C F Robinson

W M Clow

\section{Questions}

1 What do the figures show?

2 What two major complications of these problems may be expected?

3 What management priorities would you set?

Accepted 24 October 1997

4 How would you achieve these objectives? 


\section{Answers}

QUESTION 1

The inferior cavagram shows complete occlusion of the inferior vena cava up to and beyond the renal veins. Co-lateral flow only is seen through the para-vertebral venous plexus. Dynamic imaging suggested that the occlusion was due to thrombus in association with extrinsic compression.

The two selected cuts from the abdominal CT scan demonstrate a huge homogenous unilocular cyst, arising from the left ovary and extending up to the epigastrium, flattening the inferior vena cava and the left common iliac vein.

QUESTION 2

The two major complications are pulmonary emboli and bilateral renal vein occlusion leading to nephrotic syndrome and/or renal failure.

QUESTION 3

There are four major management objectives: restoration of venous patency in the inferior vena cava, prevention of pulmonary emboli, removal of the ovarian cyst, and determination of any underlying predisposing pathologies.

\section{QUESTION 4}

Treatment is urgent to prevent further clot extension and to re-establish venous patency. Heparin followed by oral anticoagulation helps prevent extension of the thrombus and reduces the risk of pulmonary embolism. Thrombolysis, however, may restore venous patency, decrease the likelihood and severity of the post-phlebitic syndrome, and reduce the risk of major pulmonary embolism. ${ }^{1}$ Unfortunately, thrombolysis itself may cause pulmonary embolisation secondary to clot fragmentation, ${ }^{2}$ and temporary caval filtration is advisable. Local catheter-directed thrombolysis is superior to systemic thrombolysis, and is the treatment of choice in extensive proximal venous thrombosis. $^{34}$

Caval compression and deep vein thrombosis are both recognised complications of large ovarian cysts. The most likely reason in this patient is mechanical obstruction due to external caval compression by the cyst. Given the unusual site and extensive nature of the thrombosis, other causes of thrombophilia must also be considered, including possible malignancy of the cyst, a functioning ovarian tumour, the

1 Armonson MP, Hopkinson BR. Thrombolysis for acute deep vein thrombosis. Br $\mathcal{F}$ Surg 1996;83:580-1.

2 Goldsmith JC, Lollar P, Hoak JC. Massive fatal pulmonary embolism with fibrinolytic therapy. Circulation 1981;64: 1068-9.

3 Semba CP, Drake MD. Ilio-femoral deep vein thrombosis aggressive therapy with catheter directed thrombolysis Radiology 1994;191:487-94. oral contraceptive pill and other rarer causes of inherited and acquired thrombophilia.

\section{Management}

A thrombophilia screen and $\mathrm{Ca} 125$ were negative, and detailed imaging suggested that the ovarian cyst was benign. An early lung scan showed several small perfusion defects in both lung fields, despite treatment with heparin. The extensive nature of the thrombus beyond the renal veins precluded early insertion of a caval filter, so we opted for primary thrombolysis. Streptokinase, tissue plasminogen activator, and urokinase have all been used for caval thrombolysis, but there has been debate as to which agent is preferable, and what dose to utilise. $^{5}$ We used streptokinase (250 000 units over $30 \mathrm{~min}$ followed by an infusion of 50000 units hourly) infused via a catheter inserted into the right femoral vein and advanced to the origin of the inferior vena cava. The response to this initial agent was poor (perhaps because of lack of clot specificity, or streptococcal antibodies), but the response to tissue plasminogen activator (infused at $4 \mathrm{mg} / \mathrm{h}$ ) was dramatic. Once the majority of fresh thrombus had cleared, the patient was taken to the operating theatre where a Gunther temporary vena caval filter (William Cook Company, Denmark) was inserted via the right internal jugular vein to lie just below the renal veins. We considered filter insertion prior to decompression of the ovarian cyst to be extremely important since this would be a high-risk period for thrombus migration. Laparotomy demonstrated a huge benign serous cystadenoma which was decompressed (7.3 litres) and removed with the left ovary. A postoperative cavagram showed a clear inferior vena cava and the temporary filter was removed. Long-term anticoagulation with warfarin was initiated.

At a six-month review, there was very little post-phlebitic swelling of the left leg and no pain. Renal function was normal, and the chest X-ray clear.

\section{Final diagnosis}

Complete occlusion of the inferior vena cava resulting from a large unilocular ovarian cyst.

Keywords: ovarian cyst; caval compression; deep vein thrombosis

4 Comerota AJ, Aldridge SC. Thrombotic therapy for deep vein thrombosis:a clinical review. Can $\mathcal{F}$ Surg 1993;36:359 64.

5 Turpie AGG. Thrombolytic therapy in venous thrombosis and pulmonary embolism. Fibrinolysis 1994;8 (suppl 1) 237-44. 